\title{
Effect of co-culture with amniotic epithelial cells on the biological characteristics of amniotic mesenchymal stem cells
}

\author{
LI-JING RAN ${ }^{1,2}$, YUN ZENG ${ }^{1}$, SHAO-CHUN WANG ${ }^{2}$, DI-SI ZHANG ${ }^{1}$, \\ MIN HONG ${ }^{3}$, SHAO-YOU LI ${ }^{3}$, JIAN DONG ${ }^{3}$ and MING-XIA SHI ${ }^{1}$
}

\author{
${ }^{1}$ Department of Hematology, The First Affiliated Hospital of Kunming Medical University, \\ Hematology Research Center of Yunnan Province, Kunming, Yunnan 650032; ${ }^{2}$ Department of Ultrasonography, \\ The Affiliated Hospital of Jining Medical College, Jining, Shandong 272000; ${ }^{3}$ Department of Oncology, \\ The First Affiliated Hospital of Kunming Medical University, Kunming, Yunnan 650032, P.R. China
}

Received March 3, 2017; Accepted February 9, 2018

DOI: $10.3892 / \mathrm{mmr} .2018 .9053$

\begin{abstract}
The aim of the present study was to investigate the effect of co-culture with amniotic epithelial cells (AECs) on the biological characteristics of amniotic mesenchymal stem cells (AMSCs), to compare the expression of $\mathrm{C}-\mathrm{X}-\mathrm{C}$ motif chemokine receptor 4 (CXCR4) in co-cultured AMSCs and to investigate the roles of the stromal cell-derived factor-1 (SDF-1)/CXCR4 axis in the homing and migration of AMSCs. AMSCs were isolated from human amniotic membranes, purified and then differentiated into osteoblasts and adipocytes in vitro, which was verified by von Kossa Staining and Oil Red O staining. Cell viability was measured by Cell Counting kit- 8 and trypan blue assays at 24,48 and $72 \mathrm{~h}$, the expression of CXCR4 was analyzed by immunofluorescence-based flow cytometry and reverse transcription-quantitative polymerase chain reaction, and the migration ability of AMSCs in vitro was observed by a migration assay. The results demonstrated that cell viability (at 48 and $72 \mathrm{~h}$ ) and survival (at 24, 48 and $72 \mathrm{~h}$ ) in the co-culture and serum groups were higher compared with the serum-free group. Furthermore, CXCR4 mRNA and protein expression, and migration along the SDF-1 gradient, in the co-culture and serum-free groups were higher compared with the serum group. Overall, the results indicated that AMSCs co-cultured with AECs exhibited enhanced
\end{abstract}

Correspondence to: Dr Ming-Xia Shi, Department of Hematology, The First Affiliated Hospital of Kunming Medical University, Hematology Research Center of Yunnan Province, 295 Xichang Road, Kunming, Yunnan 650032, P.R. China

E-mail: mingxiashi@126.com

Abbreviations: AECs, amniotic epithelial cells; MSCs, mesenchymal stem cells; AMSCs, amniotic mesenchymal stem cells; CXCR4, C-X-C motif chemokine receptor 4; GVHD, graft versus host disease; BMSCs, bone marrow mesenchymal stem cells; IL, interleukin

Key words: amniotic mesenchymal stem cells, amniotic epithelial cells, $\mathrm{C}-\mathrm{X}-\mathrm{C}$ motif chemokine receptor 4 , homing, migration proliferation activity and survival rate. In conclusion, the present study demonstrated that co-culture of AMSCs with AECs upregulated CXCR4 on the surface of AMSCs and enhanced the migration ability of AMSCs in vitro. This result may improve the directional migration and homing ability of AMSCs, as well as provide a theoretical basis for the application of AMSCs in clinical practice as a novel strategy to increase the success of hematopoietic stem cell transplantation.

\section{Introduction}

Mesenchymal stem cells (MSCs) are a class of pluripotent stem cells derived from the mesoderm that support the in vitro growth of long-term culture-initiating cells and promote in vivo hematopoietic embedding and reconstruction, thus having important roles in tissue repair, anti-inflammation, and the prevention and treatment of graft versus host disease (GVHD) (1,2). However, harvesting bone marrow mesenchymal stem cells (BMSCs) is invasive and their ability to differentiate decreases with age, which restricts their utility in clinical and scientific research (3). As MSCs originating from the amniotic membrane, termed amniotic MSCs (AMSCs), could be accessed relatively easily compared with BMSCs and without ethical barriers, there are numerous potential applications for AMSCs. The biological characteristics of BMSC and AMSC were previously reported to be similar, including hematopoiesis multipotency properties with low immunogenicity as well as possessing the ability to inhibit the proliferation of allogeneic T cells $(4,5)$. The combined transplantation of BMSCs with hematopoietic stem cells has been reported to be an effective method for increasing hematopoietic reconstitution and reducing the occurrence of $\operatorname{GVHD}(2,6)$. One study demonstrated that direct injection of MSCs into the bone marrow cavity promoted hematopoietic recovery and reduced GVHD symptoms (7), indicating that improving MSC homing and implantation methods may lead to improved therapeutic effects of MSC transplantation. Previous studies have also demonstrated that stimulation with a cytokine cocktail [fms-related tyrosine kinase-3 ligand, recombinant human stem cell factor, interleukin (IL)-6, hepatocyte growth factor and IL-3 increased the expression of C-X-C motif chemokine 
receptor 4 (CXCR4) on the surface of BMSCs (8) and that the stromal cell-derived factor-1 (SDF-1)/CXCR4 axis facilitated BMSC homing and accelerated hematopoietic recovery in a rat pancreatic transplant recipient (9). However, not all of the aforementioned cytokines are suitable for therapeutic use in humans, and the cytokine cocktail may induce severe adverse side effects due to their pleiotropic properties (10). Therefore, although this method is effective, it cannot be applied clinically. Determining whether there is a simpler, safer and more effective way to promote MSC homing clinically requires further investigation.

Amniotic epithelial cells (AECs) are derived from the embryonic ectoderm. These cells are able to synthetize and secrete a variety of cytokines, and have the ability to grow and proliferate in serum-free conditions $(11,12)$. Therefore, we hypothesized that co-culture of AMSCs with AECs may maintain AMSC activity and also stimulate the expression of CXCR4 on AMSC surfaces to enhance AMSC migration and homing ability.

In the current study, the effects of co-culture with AECs on the biological characteristics of AMSCs, including their viability, CXCR4 expression and migration ability, as well as the roles of the SDF-1/CXCR4 axis in the migration and homing of AMSCs, were investigated.

\section{Materials and methods}

Samples and approval. Samples of human amniotic membrane were obtained from 43 healthy women aged 22-30 years that had undergone a caesarean delivery (negative in hepatitis B virus, human immunodeficiency virus and syphilis tests) from the First Affiliated Hospital of Kunming Medical University (Kunming, China). All the samples were collected between October 2012 and March 2014. The study was approved by Ethics Committee of the First Affiliated Hospital of Kunming Medical University. Written informed consent was obtained from donors for the use of amniotic membranes in this study.

Isolation, culture and identification of AMSCs. The amniotic membrane was isolated and repeatedly rinsed under aseptic conditions. Following the removal of blood clots, the amniotic membrane was cut into sections $\left(\sim 0.5-1.0 \mathrm{~mm}^{2}\right)$ and seeded onto the bottom of culture flasks. Complete Dulbecco's modified Eagle's medium (DMEM)/F12, containing $10 \%$ fetal bovine serum (FBS) and 1\% cyan-streptomycin, all of which were purchased from HyClone (GE Healthcare Life Sciences, Logan, UT, USA), was added and the flasks were cultured at $37^{\circ} \mathrm{C}$ and in $5 \% \mathrm{CO}_{2}$ and saturated humidity. When the cell density reached $80-90 \%$, the cells were passaged. P3-6 generation AMSCs were used in the experiments.

MSCs were identified as described previously $(8,13)$. Briefly, indirect immunofluorescence was performed using MSCs $\left(1 \times 10^{6}\right)$. Cells were blocked with $0.5 \%$ bovine serum albumin (BSA) and $2 \%$ normal FBS in $1 \mathrm{X} \mathrm{PBS}$ at $4^{\circ} \mathrm{C}$ for $30 \mathrm{~min}$; both serums of which were purchased from Sigma-Aldrich; Merck KGaA (Darmstadt, Germany). Following this, cells were incubated at $4^{\circ} \mathrm{C}$ for 30 min with primary mouse anti-human monoclonal antibodies against CD11a (cat. no. 301202; 1:100), CD11b (cat. no. 301302; 1:100), CD29 (cat. no. 303002; 1:100), CD31 (cat. no. 303102; 1:50), CD34 (cat. no. 343502; 1:25),
CD44 (cat. no. 338802; 1:100), CD45 (cat. no. 368502; 1:100), CD90 (cat. no. 328102; 1:100), CD105 (cat. no. 323202; 1:50), human leukocyte antigen D-related (HLA-DR; cat. no. 307602; 1:100) and pan-cytokeratins (Pan-CK; cat. no. 628602; 1:250); which were all purchased from BioLegend, Inc. (San Diego, CA, USA). Cells were then incubated with fluorescein isothiocyanate-labeled goat anti-mouse secondary antibody (cat. no. 1015-02; 1:200; Southern Biotech, Birmingham, IL, USA) at $4^{\circ} \mathrm{C}$ for $30 \mathrm{~min}$. Isotype antibodies were used as the control. MSCs were subsequently detected by flow cytometry and the results were analyzed using WinMDI 2.9 software (Scripps Research Institute, La Jolla, CA, USA).

To induce differentiation, AMSCs were inoculated into culture flasks at a density of $2-3 \times 10^{4} / \mathrm{cm}$ at $37^{\circ} \mathrm{C}$ for 3 weeks in adipocyte differentiation medium [Iscove's modifiedDulbecco's medium $(\mathrm{IMDM})+10^{-6} \mathrm{~mol} / \mathrm{l}$ dexamethasone $+0.5 \mathrm{~mol} / \mathrm{l}$ 1-methyl-3-isobutyl-xanthine $+0.1 \mathrm{~mol} / 1 \mathrm{vitamin} \mathrm{C}+100 \mathrm{U} / \mathrm{ml}$ penicillin $+100 \mu \mathrm{g} / \mathrm{ml}$ streptomycin $+10 \% \mathrm{FBS})]$, all reagents of which were purchased from Sigma Aldrich; Merck KGaA. Following this, AMSCs were fixed in ice cold $10 \%$ formalin for $10 \mathrm{~min}$ and stained with oil red $\mathrm{O}$ for $5 \mathrm{~min}$ at room temperature. Osteogenic induction was performed in Iscove's modified Dulbecco's medium containing $10 \% \mathrm{FBS}, 10^{-7} \mathrm{~mol} / 1$ dexamethasone, $10 \mathrm{~mol} / 1 \beta$-glycerophosphate, $0.05 \mathrm{~mol} / \mathrm{l}$ vitamin $\mathrm{C}, 100 \mathrm{U} / \mathrm{ml}$ penicillin and $100 \mu \mathrm{g} / \mathrm{ml}$ streptomycin (all from Sigma Aldrich, St Louis, MO, USA). A total of 3 weeks post-induction, cells were fixed with $10 \%$ formalin for $10 \mathrm{~min}$ at room temperature and then incubated in 5\% silver nitrate (American Master Tech Scientific, Inc., Lodi, CA, USA) at room temperature for $1 \mathrm{~h}$. Observation was subsequently performed using a light microscope (magnification, $\mathrm{x} 400$ ). Negative controls refer to AMSCs stained with Oil Red O or Von Kossa that had been cultured in DMEM/F12 medium without adipogenic and osteogenetic induction.

Isolation, culture and identification of AECs. The amnion tissue was digested with $0.125 \%$ trypsin (Biological Industries, Kibbutz Beit Haemek, Israel) at $37^{\circ} \mathrm{C}$ for $30-40$ min. The digested liquid was collected and filtered through a 200-mesh screen to collect the cells following centrifugation at $200 \mathrm{x} g$ for $5 \mathrm{~min}$ at room temperature. The collected cells were then cultured at $37^{\circ} \mathrm{C}$ with $5 \% \mathrm{CO}_{2}$ in complete medium (DMEM/F12 containing 10\% FBS, $100 \mathrm{U} / \mathrm{ml}$ penicillin and $100 \mu \mathrm{g} / \mathrm{ml}$ streptomycin), the reagents of which were purchased from HyClone (GE Healthcare Life Sciences). Cells were then passaged when they reached $80-90 \%$ confluence.

The AECs were prepared for the cell climbing slice assay (14), followed by fixation in $4 \%$ neutral formaldehyde, staining with hematoxylin, differentiation with $1 \%$ hydrochloric acid for 30-60 sec, re-staining with $1 \%$ aqueous ammonia for $1 \mathrm{~min}$ and eosin for $30 \mathrm{~min}$, alcohol dehydration, 5-10 min of hyalinization and mounting on a film (14). All steps were performed at room temperature. Observation was subsequently performed using a light microscope (magnification, x100).

For immunohistochemical analysis, cell climbing slices were immersed in DMEM/F12 medium and then fixed in $4 \%$ neutral formaldehyde for $15 \mathrm{~min}$ at room temperature, subsequently inactivated via incubation with $3 \% \mathrm{H}_{2} \mathrm{O}_{2}$ for $10 \mathrm{~min}$ at room temperature and then blocked with $5 \%$ goat 
serum (cat. no. 0060-01; Southern Biotech, Birmingham, AL, USA) for $30 \mathrm{~min}$ at room temperature. Following blocking, incubation was performed for $45 \mathrm{~min}$ at room temperature with a primary antibody against Pan-CK (cat. no. sc-8018; 1:100; Santa Cruz Biotechnology, Inc., Dallas, Texas, USA). As a negative control, cells were treated with PBS in the absence of primary antibodies. The cells were then incubated with goat anti-mouse IgG-Biotin secondary antibodies for $30 \mathrm{~min}$ at room temperature, which were included in the SABC kit purchased from Beijing Solarbio Science \& Technology Co., Ltd. (Beijing, China; cat. no. SA0011), according to the manufacturer's protocol. Then cells were observed under a light microscope (magnification, x200).

A direct immunofluorescence assay was performed after rupturing cell membranes and fixing the cells $\left(10^{7}\right.$ cells $\left./ \mathrm{ml}\right)$ using a Fixation/Permeabilization Solution kit (cat. no. 554714; BD Biosciences, Franklin Lakes, NJ, USA). Cells were then incubated at room temperature for $40 \mathrm{~min}$ with phycoerythrin (PE)-labeled Pan-CK antibodies (cat. no. ab52460; 1:100; Abcam, Cambridge, UK). Cells were blocked using $10 \%$ normal human serum (cat. no. 31876; Invitrogen; Thermo Fisher Scientific, Inc., Waltham, MA, USA) at room temperature for $30 \mathrm{~min}$. As a negative control, cells were treated with isotype $\mathrm{IgG}$ in the absence of the antibodies. The cells were subsequently resuspended and identified by flow cytometry. The results were analyzed using WinMDI 2.9 software.

Co-culture groups and comparison of adipogenic and osteogenic abilities. AMSCs were digested with $0.125 \%$ trypsin at $37^{\circ} \mathrm{C}$, resuspended in serum-free DMEM/F12, seeded into 6 -well plates at a density of $1 \times 10^{5}$ cells/well and placed into a Millicell chamber $(0.4 \mu \mathrm{m}$; EMD Millipore, Billerica, MA, USA). The AECs were inoculated into the small chamber at a density of $1 \times 10^{4}$ cells/well. Together, this co-culture was labeled the co-culture group. The same batch of AMSCs were digested, resuspended in serum-free DMEM/F12 medium or complete medium (DMEM/F12 with the addition of $10 \%$ FBS) and seeded into 6-well plates using the above-mentioned methods and concentrations. These AEC-free cultures, which were termed the serum-free and serum groups, respectively, were used as controls. The AMSCs were detached using $0.125 \%$ trypsin at $37^{\circ} \mathrm{C}$ and subsequently collected for use following incubation at $37^{\circ} \mathrm{C}$ for 24,48 or $72 \mathrm{~h}$ time intervals.

In order to compare the adipogenic and osteogenic abilities between the three culture groups, cells were inoculated into culture flasks at a density of $2-3 \times 10^{4}$ cells $/ \mathrm{cm}^{2}$ in adipocyte differentiation medium (IMDM $+10^{-6} \mathrm{~mol} / \mathrm{l}$ dexamethasone $+0.5 \mathrm{~mol} / 1$ 1-methyl-3-isobutyl-xanthine $+0.1 \mathrm{~mol} / 1$ vitamin $\mathrm{C}+100 \mathrm{U} / \mathrm{ml}$ penicillin $+100 \mu \mathrm{g} / \mathrm{ml}$ streptomycin $+10 \% \mathrm{FBS}$ ) at $37^{\circ} \mathrm{C}$ for 2 weeks. Osteogenic induction was performed in IMDM containing $10 \% \mathrm{FBS}, 10^{-7} \mathrm{~mol} / \mathrm{l}$ dexamethasone, $10 \mathrm{~mol} / 1 \beta$-glycerophosphate, $0.05 \mathrm{~mol} / 1$ vitamin $\mathrm{C}, 100 \mathrm{U} / \mathrm{ml}$ penicillin and $100 \mathrm{~g} / \mathrm{ml}$ streptomycin at $37^{\circ} \mathrm{C}$ for 2 weeks. Following 2 weeks of adipogenic and osteogenic differentiation, total RNA from the AMSCs in the three groups was extracted using TRIzol reagent (Invitrogen; Thermo Fisher Scientific, Inc.) and reverse-transcribed into cDNA using a PrimeScript ${ }^{\mathrm{TM}}$ RT-PCR kit (Takara Bio, Inc., Otsu, Japan) at $42^{\circ} \mathrm{C}$ for 50 min. SYBR ${ }^{\circledR}$ Premix Ex Taq ${ }^{\mathrm{TM}}$
(Takara Bio, Inc.) was used for quantitative polymerase chain reaction (qPCR). qPCR was performed using a 7500 Fast Real-Time PCR System (Applied Biosystems; Thermo Fisher Scientific, Inc.). Alkaline phosphatase (ALP) and osteopontin (OPN) were measured as osteogenic indexes, while peroxisome proliferator-activated receptor $\gamma(\operatorname{PPAR} \gamma)$ and CCAAT/enhancer-binding protein $\alpha(\mathrm{C} / \mathrm{EBP} \alpha)$ were measured as the adipogenic indexes and GAPDH was used as the internal reference. The primer sequences used were as follows: ALP (162 bp) forward, 5'-ACCATTCCCACGTCT TCACATTTG-3' and reverse, 5'-AGACATTCTCTCGTTCAC CGCC-3'; OPN (416 bp) forward, 5'-AGCCAGGACTCCATT GACTCGAAC-3' and reverse, 5'-GTTTCAGCACTCTGG TCATCCAGC-3'; C/EBP $\alpha$ (171 bp) forward, 5'-GAAGTT GGTGGAGCTGTCGG-3' and reverse, 5'-TGAGGTATGGGT CGTTGCTGA-3'; PPAR $\gamma$ (89 bp) forward, 5'-AGCCTCATG AAGAGCCTTCCA-3' and reverse, 5'-ACCCTTGCATCC TTCACAAGC-3'; and GAPDH (393 bp) forward, 5'-GTCTTC ACCACCATGGAGAAGGCT-3' and reverse, 5'-CATGCC AGTGAGCTTCCCGTTCA-3'. The reaction conditions were pre-denaturation at $90^{\circ} \mathrm{C}$ for $10 \mathrm{sec}$, followed by degeneration at $95^{\circ} \mathrm{C}$ for $5 \mathrm{sec}$, annealing and extension at $60^{\circ} \mathrm{C}$ for $60 \mathrm{sec}$, for a total of 40 cycles. The experiment was repeated three times. The results were analyzed using the $2^{-\Delta \Delta \mathrm{Cq}}$ method (15), and the expression levels of adipogenic and osteogenic indexes were compared among the three groups after 24, 48 and $72 \mathrm{~h}$ of culture. The results were expressed in terms of $2^{-\Delta \Delta \mathrm{Cq}}$ using the following formula: $\Delta \Delta \mathrm{Cq}=\Delta \mathrm{Cq}$ (co-culture group or serum-free group)- $\Delta \mathrm{Cq}$ (serum group). The difference between the co-culture (or serum-free) group and the serum group was $2^{-\Delta \Delta \mathrm{Cq}}$ times.

Comparison of AMSC viability. For the Cell Counting kit-8 (CCK-8) assay, AMSC suspensions from each group at each time point were inoculated into 96 -well plates $\left(10^{4}\right.$ cells/well $)$ and cultured overnight at $37^{\circ} \mathrm{C} .10 \%$ CCK-8 solution (Dojindo Molecular Technologies, Inc., Kumamoto, Japan) was added to each well and plates were incubated for an additional 3-4 h at $37^{\circ} \mathrm{C}$. The optical density (OD) value of each well was determined by a microplate reader.

For the trypan blue assay, AMSC suspensions from each group at each time point $\left(10^{5}\right.$ cells/well $)$ were stained with $0.4 \%$ trypan blue dye for $30-60 \mathrm{sec}$ at room temperature. Following this, cells were delivered to a hemocytometer by capillary action. The number of blue-stained cells was determined under a light microscope (magnification, x40). The following formula was used for cell counting: Survival rate $(\%)=[$ (total number of cells-number of blue-stained cells)/total number of cells] x100.

Comparison of CXCR4 expression levels. CXCR4 expression was detected using a direct immunofluorescence assay. Cells $\left(2 \times 10^{5}\right)$ were blocked via incubation with $0.5 \%$ BSA (Beijing Solarbio Science \& Technology Co., Ltd.) at $4^{\circ} \mathrm{C}$ for $30 \mathrm{~min}$. Following this, CXCR4 cell surface expression was investigated via incubation of cells with a PE-labeled mouse anti-human CXCR4 monoclonal antibody (cat. no. 12-9999-41; 1:20; eBioscience; Thermo Fisher Scientific, Inc.) at room temperature for $30 \mathrm{~min}$. To detect intracellular CXCR4, cells $\left(2 \times 10^{5}\right)$ were blocked via incubation with $0.5 \%$ BSA 
(Beijing Solarbio Science \& Technology Co., Ltd.) at $4^{\circ} \mathrm{C}$ for $30 \mathrm{~min}$ and then incubated with unlabeled CXCR4 monoclonal antibodies (cat. no. 14-9999-80; 1:20; eBioscience; Thermo Fisher Scientific, Inc.) at room temperature for $1 \mathrm{~h}$. Following the rupturing cell membranes and fixing of the lysates using the Fixation/Permeabilization Solution kit (BD Biosciences), cells were then incubated with PE-labeled CXCR4 monoclonal antibodies for $30 \mathrm{~min}$ at room temperature for staining (cat. no. 12-9999-41; 1:20; eBioscience; Thermo Fisher Scientific, Inc.). The results obtained by flow cytometry were analyzed by WinMDI 2.9 software.

The mRNA expression of CXCR4 was also investigated in cells after 24,48 and $72 \mathrm{~h}$ of culture using reverse transcription (RT)-qPCR. Total RNA was extracted and reverse transcribed into cDNA according to the aforementioned protocol, followed by amplification with SYBR Green dye and plotting of amplification curves using a qPCR instrument. The sequences of primers targeting the CXCR4 gene were forward, 5'-ACTTCA GTTTGTTGGCTGCGGC-3' and reverse, 5'-ACCGCTGGT TCTCCAGATGCG-3'. The sequences of primers targeting the internal reference (GAPDH) were forward, 5'-GAAGGT GAAGGTCGGAGTC-3' and reverse, 5'-GAAGATGGGATG GGATTTC-3'. The following reaction conditions were used: Pre-denaturation at $95^{\circ} \mathrm{C}$ for $10 \mathrm{sec}$, followed by 40 cycles of denaturation at $95^{\circ} \mathrm{C}$ for $5 \mathrm{sec}$ and annealing and elongation at $60^{\circ} \mathrm{C}$ for $40 \mathrm{sec}$. The experiment was repeated three times and the results were expressed as the $2^{-\Delta \Delta \mathrm{Cq}}$.

In vitro migration assay. The assay was performed in a Millicell chamber (EMD Millipore), with the upper chamber membrane (pore size, $12 \mu \mathrm{m}$ ) coated with fibronectin (EMD Millipore) and the lower chamber filled with different concentrations of SDF-1 (100, 200 and 300 ng/ml; PeproTech, Inc., Rocky Hill, NJ, USA) as well as DMEM/F12 medium and 0.5\% BSA (Beijing Solarbio Science \& Technology Co., Ltd.). AMSCs $\left(3 \times 10^{5}\right.$ cells $\left./ \mathrm{ml}\right)$ in DMEM/F12 medium were added to the upper chamber. The antibody blocking group represents cells that have been incubated with PE-labeled CXR4 monoclonal antibodies as aforementioned, which blocked cell surface CXCR4. After $24 \mathrm{~h}$ of culture at $37^{\circ} \mathrm{C}$, the filter was removed and stained with $0.1 \%$ crystal violet for 15-30 min at room temperature, and the number of cells that migrated to the outer surface of the membrane was counted under a light microscope (magnification, x200). The number of cells in five random fields of view of the filter was counted and the experiment was repeated three times.

Statistical analysis. SPSS package version 17.0 for Windows (SPSS, Inc., Chicago, IL, USA) was used for the statistical analysis. Experimental data are presented as the mean \pm standard deviation. Comparisons among groups were performed using one-way analysis of variance and pairwise comparisons were performed using Fisher's least significant difference test. All experiments were performed in triplicate. $\mathrm{P}<0.05$ was considered to indicate a statistically significant difference.

\section{Results}

Isolation, culture and identification of AMSCs. Consistent with our previous studies $(13,16)$, the isolated and cultured
AMSCs were spindle-shaped or polygonal, homogeneous, and transparent. Flow cytometry demonstrated that CD29, CD44, CD90 and CD105 expression was observed in AMSCs, but there was no or limited expression of CD11a, CD11b, CD31, CD34, CD45, HLA-DR and Pan-CK (data not shown). Additionally, the cells successfully differentiated into adipocytes and osteoblasts following induction in vitro (data not shown), which is consistent with other recent reports (17-19).

Isolation, culture and identification of AECs. The AECs were polygonal or oval, with a clear outline, rich cytoplasm and pavement-like appearance following growing in flakes or clusters. Hematoxylin and eosin staining demonstrated abundant cytoplasm and blue-stained nuclei (Fig. 1A). The expression of Pan-CK in AECs was observed by immunohistochemistry (Fig. 1B and C) and flow cytometry (Fig. 1D-F), both of which indicated a high degree of positive Pan-CK expression in AECs. Pan-CK represents the main structural protein and differentiation marker of the epithelium (12). The immunofluorescence staining results revealed a high expression of Pan-CK in AEC, as well as positive Pan-CK revealed by immunohistochemistry. Considering this as well as the morphological characteristics revealed, it was confirmed that the cultured cells were epithelial cells.

Basic biological characteristics of AMSCs. The AMSCs in the three groups exhibited no marked alterations in morphology after 24,48 or 72 h of culture; the morphology of AMSCs in the co-cultured group, serum-free cultured group and serum cultured group at $72 \mathrm{~h}$ are presented in Fig. 2A-C, respectively. Following co-culture with AECs for $72 \mathrm{~h}$, AMSCs also maintained stable immunophenotypic features, including a highly expressed matrix and stromal cell antigen (CD29, CD44, CD105 and CD90), and no exhibition of hematopoietic cell markers (CD11a, CD11b, CD34 and CD45), major histocompatibility antigen complex class II molecules (HLA-DR), or epithelial Pan-CK and endothelial markers (CD31) (Fig. 2D-N). M1 indicates cells with negative expression and M2 indicates cells with positive expression. Furthermore, oil red $\mathrm{O}$ and Von Kossa staining confirmed that AMSCs co-cultured with AECs for $72 \mathrm{~h}$ were able to differentiate into adipocytes and osteoblasts in vitro (Fig. 3A-D). Additionally, RT-qPCR was performed to measure the mRNA expression of osteogenic (ALP and OPN) and adipogenic $(\mathrm{C} / \mathrm{EBP} \alpha$ and PPAR $\gamma)$ markers in AMSCs following co-culture with AECs, and the results presented in Fig. 3E-H confirmed that the osteogenic and adipogenic differentiation potential of AMSCs co-cultured with AECs for $72 \mathrm{~h}$ was not altered compared with serum-free and serum cultured control groups at the same time-point.

Comparison of AMSC viability. The results of the CCK-8 assay demonstrated that no significant differences were observed in the viability among the three groups after $24 \mathrm{~h}$ of culture. However, after 48 and $72 \mathrm{~h}$ of culture, the absorbance of the co-culture and serum groups was significantly higher compared with the serum-free cultured group $(\mathrm{P}<0.05)$, indicating that the viability of AMSCs was higher in the co-culture and serum cultured groups (Fig. 4A). 


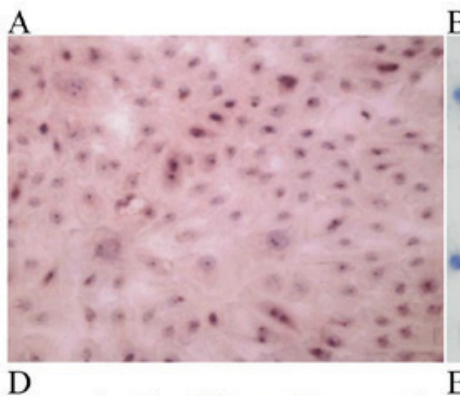

B

$\mathrm{C}$
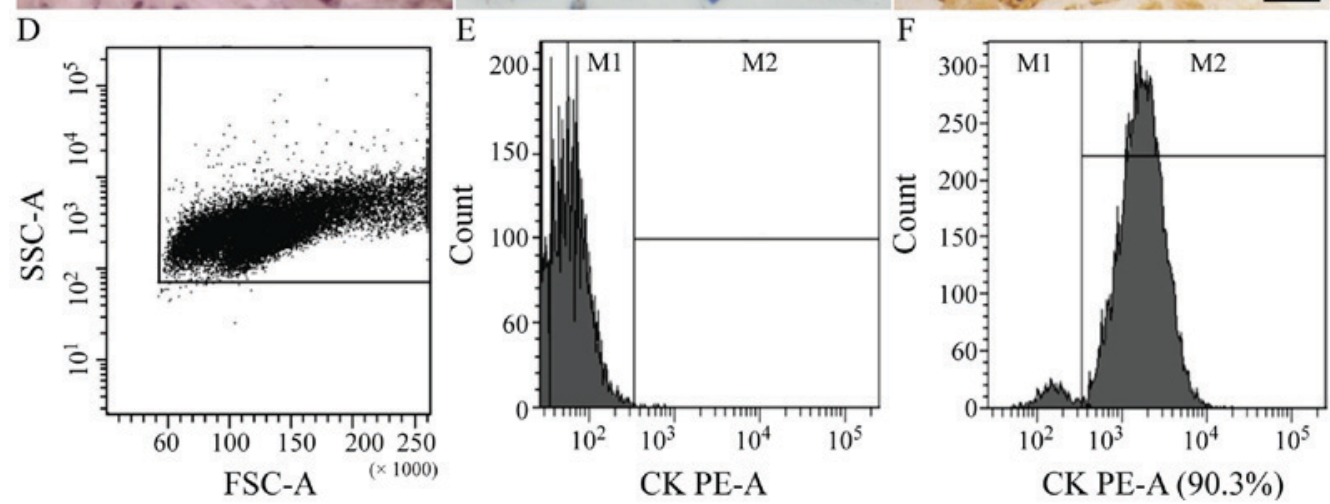

Figure 1. Identification of AECs. (A) Morphology of AECs by hematoxylin and eosin staining. Magnification, x100. (B) Negative control for Pan-CK immunohistochemical staining. Magnification, x200. (C) Immunohistochemical staining for Pan-CK indicated positive expression in AECs. (D) Flow cytometry scatter plot of AECs. (E) Negative control for Pan-CK immunofluorescence and flow cytometry experiment. (F) Flow cytometry following immunofluorescence staining indicated a high expression of Pan-CK in AECs. M1 indicates negative cells and M2 indicates positive cells. AECs, amniotic epithelial cells; Pan-CK, pan cytokeratin; SSC, side scatter; FSC, forward scatter; PE, phycoerythrin.

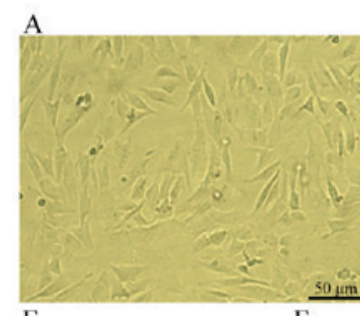

B

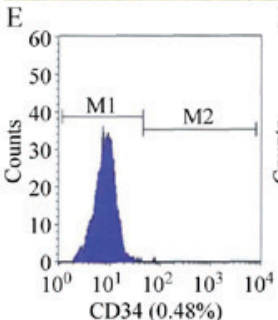

J

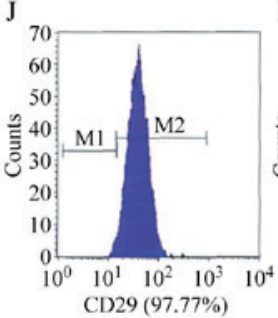

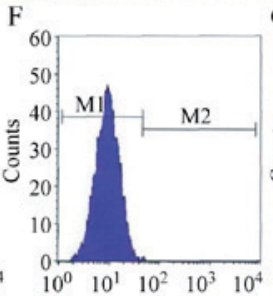
CD45 (1.34\%)

K

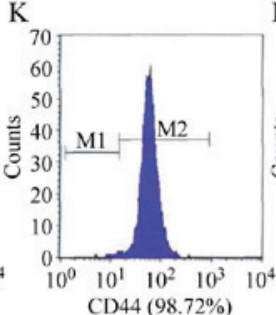

C
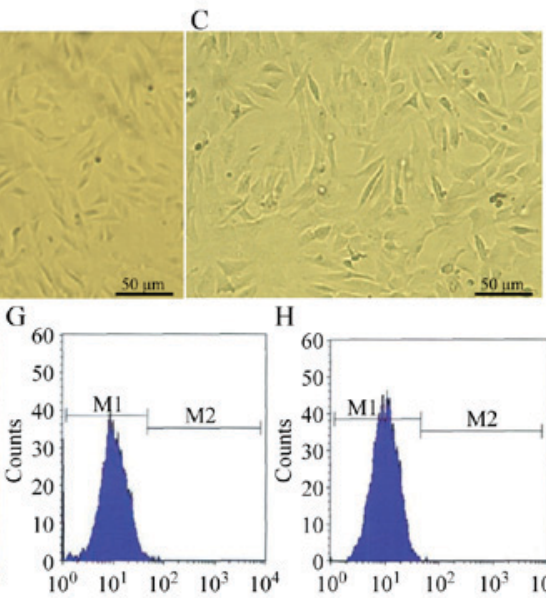

CD31 $(0.75 \%)$

L

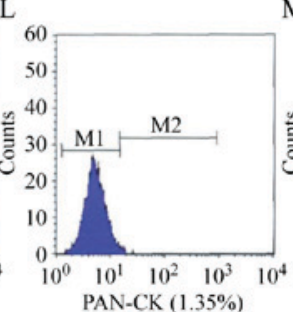

H

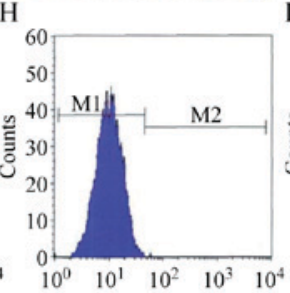

CDIIb $(0.26 \%)$

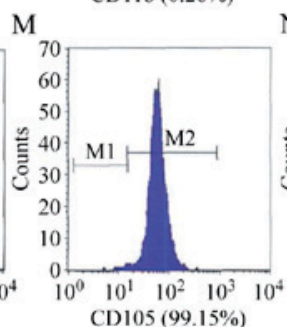

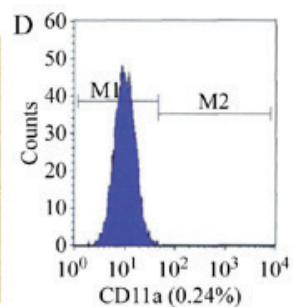

I

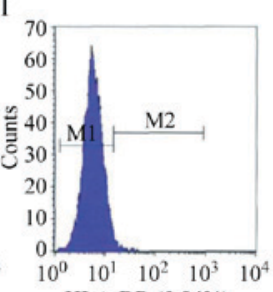

N

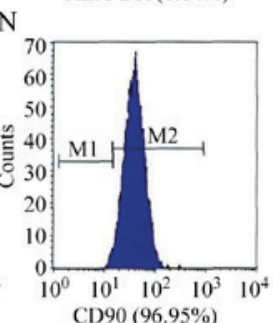

Figure 2. Biological characteristics of AMSCs following co-culture with AECs. The morphology of AMSCs at $72 \mathrm{~h}$ in the (A) co-cultured group, (B) serum-free cultured group and (C) serum cultured group. Magnification, x40. Phenotype analysis of culture-expanded AMSCs following co-culture with AECs for 72 h. The expression of (D) CD11a, (E) CD34, (F) CD45, (G) CD31, (H) CD11b, (I) HLA-DR, (J) CD29, (K) CD44, (L) Pan-CK, (M) CD105 and (N) CD90 was measured using fluorescence-labeled antibody staining and flow cytometry. The graph outlined the region of fluorescent intensity for cells fluorescently labeled with primary antibodies for different markers. M1 indicates negative cells and M2 indicates positive cells. AMSCs, amniotic mesenchymal stem cells; AECs, amniotic epithelial cells; HLA-DR, human leukocyte antigen D-related; Pan-CK, pan cytokeratins.

Furthermore, trypan blue staining demonstrated that the survival rates of AMSCs in the co-culture and serum groups at all three time-points were significantly higher compared with AMSCs in the serum-free group ( $\mathrm{P}<0.05$; Fig. 4B). 


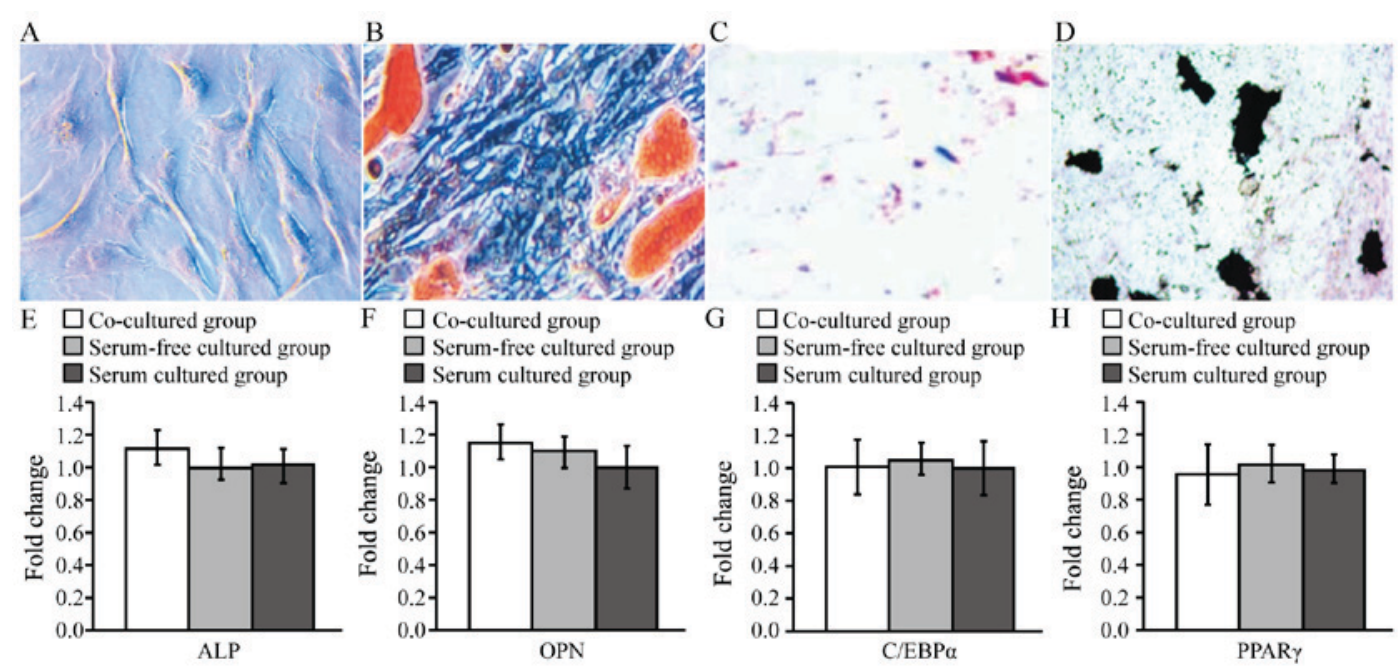

Figure 3. Adipogenic and osteogenic differentiation of AMSCs following co-culture with AECs. (A) Negative control for oil red O staining at $72 \mathrm{~h}$ following co-culture of AMSCs with AECs. (B) AMSCs co-cultured with AECs for $72 \mathrm{~h}$ exhibited positive oil red O staining, indicating successful adipogenic differentiation. (C) Negative control for Von Kossa staining at $72 \mathrm{~h}$ following co-culture of AMSCs with AECs. (D) Von Kossa staining demonstrated that AMSCs co-cultured with AECs for $72 \mathrm{~h}$ exhibited positive staining for calcium accumulation following osteogenic induction for 3 weeks. Magnification, $\mathrm{x} 400$. Reverse transcription-quantitative polymerase chain reaction demonstrated that there were no significant differences in the mRNA expression of (E) ALP and (F) OPN osteogenic markers or $(\mathrm{G}) \mathrm{C} / \mathrm{EBP} \alpha$ and $(\mathrm{H})$ PPAR $\gamma$ adipogenic markers among the three groups at $72 \mathrm{~h}$ of culture. AMSCs, amniotic mesenchymal stem cells; AECs, amniotic epithelial cells; ALP, alkaline phosphatase; OPN, osteopontin; C/EBP $\alpha$, CCAAT/enhancer-binding protein $\alpha$; PPAR $\gamma$, peroxisome proliferator-activated receptor $\gamma$.
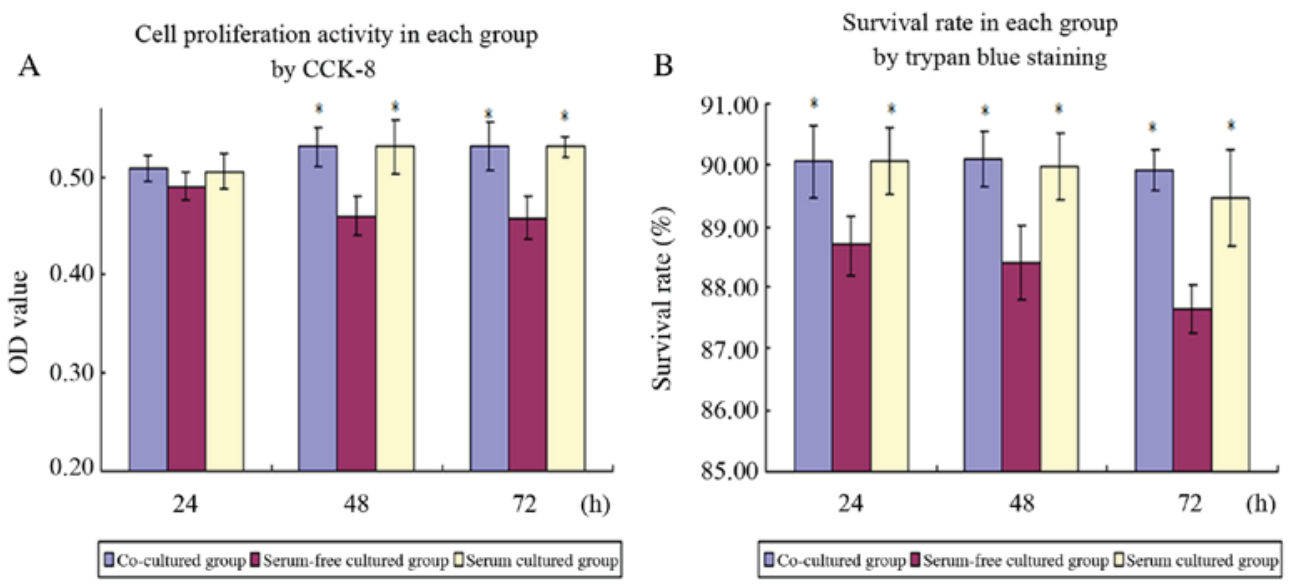

Figure 4. The effect of AMSC co-culture with amniotic epithelial cells on the cell viability of AMSCs were detected by CCK-8 assays and trypan blue staining. (A) Proliferation activity in each group was measured using a CCK-8 assay and expressed as an OD value The OD values of the co-cultured group and serum cultured group were significantly higher compared with the serum-free cultured group at 48 and $72 \mathrm{~h}$. (B) Trypan blue staining was performed to assess the survival rate of different groups. The survival rates of the co-cultured group and serum cultured group were higher compared with the serum-free cultured group at 24,48 and $72 \mathrm{~h}$ of culture. " $\mathrm{P}<0.05$ vs. serum-free cultured group at same time-point. AMSCs, amniotic mesenchymal stem cells; CCK-8, Cell Counting kit-8; OD, optical density.

CXCR4 expression. CXCR4 expression was initially measured using a direct immunofluorescence and flow cytometry assay. The results demonstrated that CXCR4 expression on cell surfaces in the co-culture and serum-free groups was higher compared with the serum cultured group at each of the three time points $(\mathrm{P}<0.05$; Fig. 5A-D). Intracellular CXCR4 expression in the co-culture group was significantly higher compared with the other two groups at $24 \mathrm{~h}(\mathrm{P}<0.05)$, but the expression of CXCR4 among the three groups at 48 and $72 \mathrm{~h}$ was not significantly different (Fig. 5B and D).

CXCR4 mRNA expression was also measured in AMSCs by RT-qPCR. The results indicated that the mRNA expression of CXCR4 at $24 \mathrm{~h}$ was $1.664 \pm 0.288$ and $1.227 \pm 0.289$ times higher in the co-culture and serum-free groups, respectively, compared with the serum group. At $48 \mathrm{~h}$, the levels of CXCR4 expression were $2.875 \pm 0.260$ and $2.842 \pm 0.413$ times greater, respectively, and at $72 \mathrm{~h}$, these levels were $3.241 \pm 0.511$ and $2.998 \pm 0.632$ times greater, respectively $(\mathrm{P}<0.05$; Fig. $5 \mathrm{E}-\mathrm{G})$.

Migration assay. The results presented in Fig. 6A and B indicate that after $48 \mathrm{~h}$ of culture in the co-culture group, the number of migrating AMSCs was increased when $200 \mathrm{ng} / \mathrm{ml}$ SDF-1 was added compared with the addition of $0 \mathrm{ng} / \mathrm{ml}$ SDF-1. The results of the migration assays also demonstrated that, in all three groups, cells migrated towards SDF-1 in a dose-dependent manner. Compared with AMSCs in the 

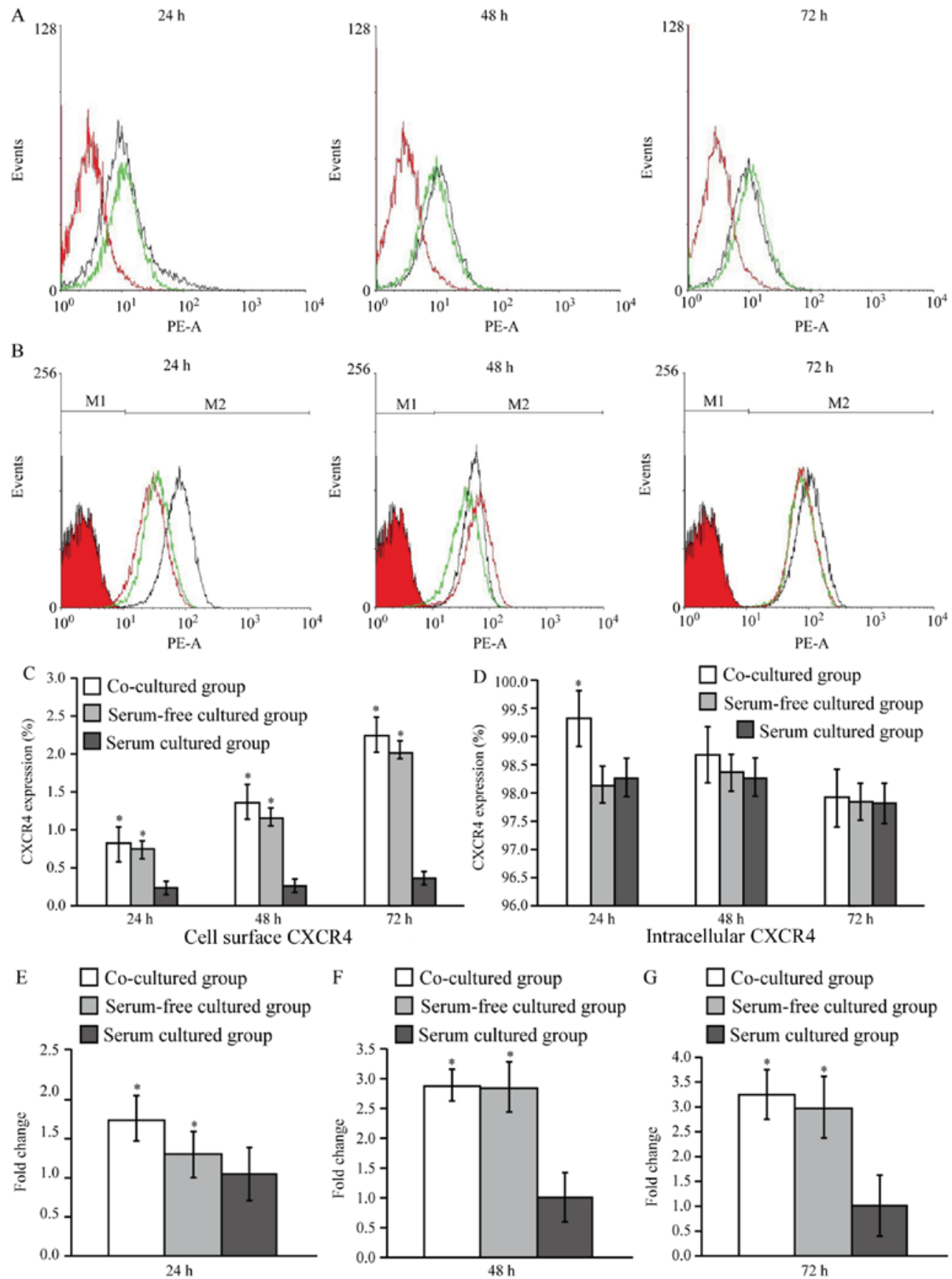

Figure 5. Expression of CXCR4 in AMSCs co-cultured with amniotic epithelial cells. (A) Cell surface CXCR4 expression of AMSCs in the co-cultured group and serum-free cultured group was higher compared with the serum cultured group. (B) Intracellular CXCR4 expression of AMSCs in the co-cultured group was significantly higher compared with the serum-free cultured group and serum cultured group at $24 \mathrm{~h}$, while no significant differences were observed among the three groups at 48 and $72 \mathrm{~h}$. Black, green and red curves represent the co-cultured, serum-free cultured and serum cultured groups, respectively. The red filled area of the graphs indicates the region of fluorescent intensity for cells labeled with isotype control antibodies. M1 indicates cells with negative expression and M2 indicates cells with positive expression. Quantification of the expression levels of (C) cell surface and (D) intracellular CXCR4 by flow cytometry. (E-G) Reverse transcription-quantitative polymerase chain reaction results demonstrated that the mRNA expression of CXCR4 was higher in the co-cultured and serum-free cultured groups compared with the serum cultured group. Values in the co-cultured and serum-free cultured groups were calculated relative to the serum cultured group. Data are presented as the mean \pm standard error of the mean of three independent experiments in triplicate. "P $<0.05$ vs. serum cultured group. CXCR4, C-X-C motif chemokine receptor 4; AMSCs, amniotic mesenchymal stem cells; PE, phycoerythrin.

serum group, the migration of AMSCs in the co-culture and serum-free groups was significantly higher at concentrations of 100,200 and $300 \mathrm{ng} / \mathrm{ml}$ at all time-points $(\mathrm{P}<0.05)$, but there were no significant differences between the co-culture and serum-free groups (Fig. 6C-E). Pre-incubation with AMSC-neutralizing antibodies prevented migration, which confirmed the specificity of this migration (Fig. 6C-E).

\section{Discussion}

MSCs have been used in the field of stem cell transplantation due to their multi-directional differentiation potential and ability to regulate immune responses (20). The number of homing MSCs is reported to be closely associated with treatment outcomes, whereas outcomes are not positively associated 


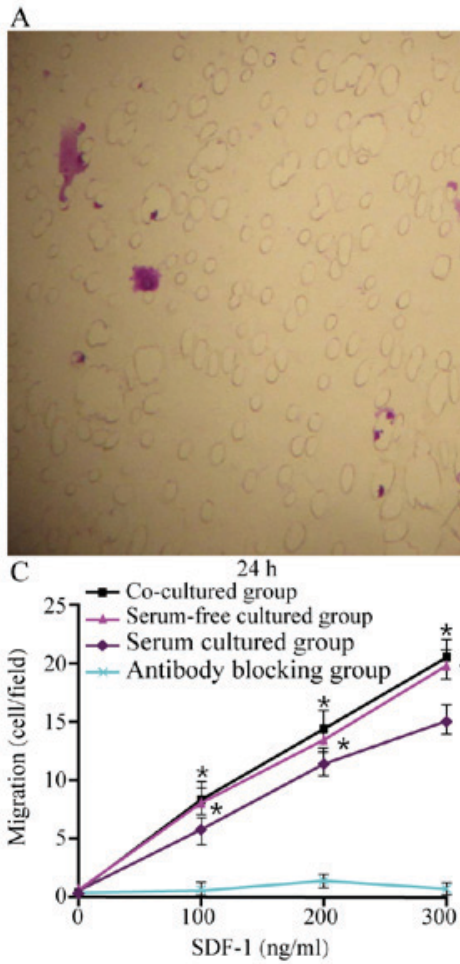

$\mathrm{B}$
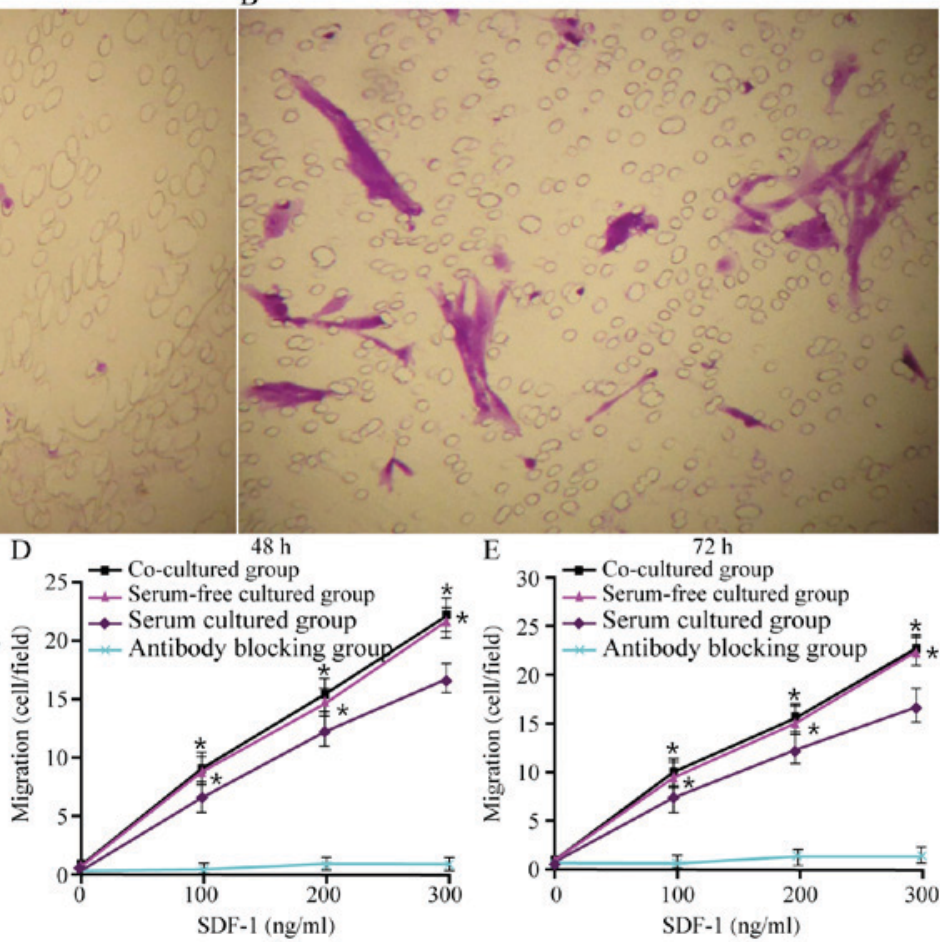

Figure 6. Migration of AMSCs co-cultured with amniotic epithelial cells along a SDF-1 gradient. A migration assay was performed to assess the migration of AMSCs in each group along a SDF-1 gradient after 24, 48 and $72 \mathrm{~h}$ of culture. Representative images of crystal violent-stained cells in the lower chamber of the co-cultured group following the addition of (A) $0 \mathrm{ng} / \mathrm{ml}$ and (B) $200 \mathrm{ng} / \mathrm{ml}$ SDF-1 after $48 \mathrm{~h}$ of culture. Magnification, x200. (C-E) Migratory cells/field were measured in five randomly selected fields for each group. After 24, 48 and $72 \mathrm{~h}$ of culture, the migration of AMSCs towards various doses of SDF-1 in the co-culture and serum-free culture groups was higher compared with the serum cultured group. ${ }^{\text {" }} \mathrm{P}<0.05$ vs. serum cultured group at a concentration of $300 \mathrm{ng} / \mathrm{ml}$ SDF-1. AMSCs, amniotic mesenchymal stem cells; SDF-1, stromal cell-derived factor-1.

with the number of MSCs transplanted (21). Therefore, the efficiency of MSC homing and target tissue implantation is key in effective treatment. The SDF-1/CXCR4 axis has been reported to have an important role in MSC homing (22). Increasing the expression of CXCR4 contributed to the migration of MSCs toward target organs and, if receptors were blocked, this ability was reduced (23).

The clinical application of BMSCs has been limited by few donors and an invasive method of obtaining them, while AMSCs are abundantly available as a by-product of childbirth and exhibit similar biological characteristics to BMSCs (24), indicating that they may have potential for numerous applications. Due to the ability of AECs to secrete various cytokines, the present study co-cultured AECs with AMSCs under serum-free conditions, aiming to maintain the growth activity of AMSCs and upregulate CXCR4, thus improving the homing and migration abilities of AMSCs. AMSCs were incubated with AECs in serum-free medium through a Millicell chamber, in which only active substances secreted by the cells were available to meet the nutritional requirements of the cells and the effects of cell contact were excluded. The results demonstrated that the co-cultured AMSCs were not different in morphology, immunophenotype or multidirectional differentiation ability when compared with those without co-culture. Furthermore, the co-cultured cells exhibited a similar growth ability compared with the serum cultured group, and this ability was superior to that in the serum-free cultured group, indicating that the cytokines produced by the AECs were sufficient to maintain the biological activity of AMSCs for at least $72 \mathrm{~h}$.
CXCR4 is primarily expressed in the cytoplasm, and cells regulate the expression of CXCR4 on cell membrane surfaces through endocytosis (25). Under normal circumstances, only a very small proportion of CXCR4 is expressed on the surface of MSCs (1-3.9\%); however, following the rupture of membranes and exposure of intracellular antigens, CXCR4 expression is reported to increase (26). Li et al (27) demonstrated that CXCR4 expression on the surface of MSCs was minimal, consistent with the results of the immunofluorescence assay in the present study. Li et al (27) also revealed that the expression of CXCR4 within cells is always higher ( $>95 \%)$ than the cell surface expression $(<5 \%)$, regardless of the method of detection used. Studies have indicated that the expression of CXCR4 on the cell surface maybe regulated by externalization and endocytosis $(25,28,29)$.

Therefore, it maybe hypothesized that co-culture of AECs and AMSCs promotes the expression of CXCR4 in AMSCs through autocrine or paracrine secretion in a serum-free environment. These cytokines may even promote the migration of intracellular CXCR4 to the cell surface. Notably, it was observed in the present study that in serum-free conditions, CXCR4 was upregulated on cell surfaces, which may explain why, even in the absence of nutritional supplements, AMSCs are able to secrete certain cytokines in an autocrine manner to maintain their growth requirements, and these cytokines may also increase the surface expression of CXCR4. However, these limited cytokines cannot meet the requirements for cell growth and proliferation, which may explain why, despite CXCR4 expression in the serum-free group being 
higher compared with the co-culture and serum groups, cell viability was lower in the serum-free group compared with other groups. Conversely, although the serum cultured group did not appear to have impaired cell viability, its CXCR4 expression was significantly lower compared with the other two groups. Therefore, the AMSCs co-cultured with AECs had the advantages of both upregulated CXCR4 and increased cell viability, while the other two groups had only one of these advantages. Although the serum-free cultured cells exhibited a similar migratory ability to the co-cultured group, the proliferation activity and survival rate of the cells were suppressed. However, further studies are required in order to confirm the above findings.

The effectiveness of chemokine receptors depends on their expression on cell surfaces. In our previous study (8), BMSCs were treated with five cytokines that upregulated the expression of CXCR4 on and within the cells, which enhanced their ability to migrate towards SDF-1, and promoted their ability to home towards bone marrow and be successfully implanted in radiated NOD/SCID mice. CXCR4-expressing MSCs have also be reported to migrate towards target organs or tissues along an SDF-1 concentration gradient and to participate in tissue repair (27,30). The migration assay performed in the current study demonstrated that the in vitro migration ability of the co-culture group along the SDF-1 concentration gradient was increased, potentially in response to the increased expression of CXCR4 on cell surfaces. Pre-incubation with AMSC-neutralizing antibodies to block surface CXCR4 prevented migration and thus confirmed that the expression of chemotactic receptors was on cell surfaces rather than intracellular, and that surface CXCR4 represents the main factor affecting migration.

There were a number of limitations associated with the presents study. Although the current study has discussed the biological characteristics of AMSCs co-cultured with AECs; however, further studies are required to investigate the biological characteristics of BMSCs co-cultured with AECs. Numerous studies have revealed that the CXCR4 expression on cell surface can be regulated by externalization and endocytosis $(28,29)$. In the present study, the results of the intracellular expression levels of CXCR4 were not entirely consistent with the results demonstrating the expression levels of CXCR4 on the cell surface. Therefore, it can be hypothesized that AECs and AMSCs upregulate the expression of CXCR4 via autocrine and paracrine secretion, respectively, in a serum-free environment. Such cytokines may also promote the migration of intracellular CXCR4 to the cell surface. However, such hypotheses require further investigation by future studies.

In conclusion, the results of the present study indicated that co-culturing AMSCs with AECs upregulated CXCR4 expression on the surfaces of AMSCs and improved the ability of AMSCs to migrate along an SDF-1 gradient. These results may set the foundation for improving the directional migration and homing ability of AMSCs, and also provide a reliable theoretical basis for the application of AMSCs in clinical practice as a novel strategy to increase the success of hematopoietic stem cell transplantation.

\section{Acknowledgements}

Not applicable.

\section{Funding}

The present study was supported by the National Natural Science Foundation of China (grant no. 31260232), the Research Institute Projects of Medical Research Units of Yunnan (grant no. 2016NS048), the Training Program of Medical Discipline Leader in Yunnan Province (grant no. 2010CI013) and the Joint Fund of Department of Yunnan Provincial Science and Technology-Kunming Medical University (grant no. 2017FE468-030).

\section{Availability of data and materials}

The datasets used and/or analyzed during the current study are available from the corresponding author on reasonable request.

\section{Authors' contributions}

MXS, YZ and SCW designed the present study; LJR and DSZ collected the data; LJR performed the statistical analysis; DSZ, JD, MH and SYL interpreted the data; LJR wrote and revised the manuscript; MXS and JD revised the manuscript for important intellectual content.

\section{Ethics approval and consent to participate}

The present study was approved by the Ethics Committee of the First Affiliated Hospital of Kunming Medical University and written informed consent was obtained from donors for the use of human amniotic membranes.

\section{Consent for publication}

Not applicable.

\section{Competing interests}

The authors declare that they have no competing interests.

\section{References}

1. Castro-Manrreza ME and Montesinos JJ: Immunoregulation by mesenchymal stem cells: Biological aspects and clinical applications. J Immunol Res 2015: 394917, 2015

2. Bernardo ME and Locatelli F: Mesenchymal stromal cells in hematopoietic stem cell transplantation. Methods Mol Biol 1416: 3-20, 2016.

3. Larsen S and Lewis ID: Potential therapeutic applications of mesenchymal stromal cells. Pathology 43: 592-604, 2011.

4. Manuelpillai U, Moodley Y, Borlongan CV and Parolini O: Amniotic membrane and amniotic cells: Potential therapeutic tools to combat tissue inflammation and fibrosis? Placenta 32 (Suppl 4): S320-S325, 2011.

5. Shi MX, Fang BJ,Liao LM, Yang SG, Liu YH and Zhao CH: Flk1+ mesenchymal stem cells ameliorate carbon tetrachloride-induced liver fibrosis in mice. Sheng Wu Gong Cheng Xue Bao 21: 396-401, 2005 (In Chinese).

6. Wu Y, Wang Z, Cao Y, Xu L, Li X, Liu P, Yan P, Liu Z, Zhao D, Wang J, et al: Cotransplantation of haploidentical hematopoietic and umbilical cord mesenchymal stem cells with a myeloablative regimen for refractory/relapsed hematologic malignancy. Ann Hematol 92: 1675-1684, 2013

7. Nakamura K, Inaba M, Sugiura K, Yoshimura T, Kwon AH, Kamiyama Y and Ikehara S: Enhancement of allogeneic hematopoietic stem cell engraftment and prevention of GVHD by intra-bone marrow bone marrow transplantation plus donor lymphocyte infusion. Stem Cells 22: 125-134, 2004. 
8. Shi M, Li J, Liao L, Chen B, Li B, Chen L, Jia H and Zhao RC: Regulation of CXCR4 expression in human mesenchymal stem cells by cytokine treatment: Role in homing efficiency in NOD/SCID mice. Haematologica 92: 897-904, 2007.

9. Gong J, Meng HB, Hua J, Song ZS, He ZG, Zhou B and Qian MP. The SDF-1/CXCR4 axis regulates migration of transplanted bone marrow mesenchymal stem cells towards the pancreas in rats with acute pancreatitis. Mol Med Rep 9: 1575-1582, 2014

10. Rider P, Carmi Y and Cohen I: Biologics for targeting inflammatory cytokines, clinical uses, and limitations. Int J Cell Biol 2016: 9259646, 2016.

11. Yang S, Sun HM, Yan JH, Xue H, Wu B, Dong F, Li WS, Ji FQ and Zhou DS: Conditioned medium from human amniotic epithelial cells may induce the differentiation of human umbilical cord blood mesenchymal stem cells into dopaminergic neuron-like cells. J Neurosci Res 91: 978-986, 2013.

12. Tabatabaei M, Mosaffa N, Nikoo S, Bozorgmehr M, Ghods R, Kazemnejad S, Rezania S, Keshavarzi B, Arefi S, Ramezani-Tehrani $\mathrm{F}$, et al: Isolation and partial characterization of human amniotic epithelial cells: The effect of trypsin. Avicenna J Med Biotechnol 6: 10-20, 2014.

13. Fang B, Shi M, Liao L, Yang S, Liu Y and Zhao RC: Multiorgan engraftment and multilineage differentiation by human fetal bone marrow Flk1+/CD31-/CD34-Progenitors. J Hematother Stem Cell Res 12: 603-613, 2003.

14. Sawa M, Inoue M, Yabuki A, Kohyama M, Miyoshi N, Setoguchi A and Yamato O: Rapid immunocytochemistry for the detection of cytokeratin and vimentin: Assessment of its diagnostic value in neoplastic diseases of dogs. J Vet Clin Pathol 46: 172-178, 2017.

15. Livak KJ and Schmittgen TD: Analysis of relative gene expression data using real-time quantitative PCR and the 2(-Delta Delta C(T)) method. Methods 25: 402-408, 2001.

16. Shi M, Li W, Li B, Li J and Zhao C: Multipotency of adult stem cells derived from human amnion. Sheng Wu Gong Cheng Xue Bao 25: 754-760, 2009 (In Chinese).

17. Vojdani Z, Babaei A, Vasaghi A, Habibagahi M and Talaei-Khozani T: The effect of amniotic membrane extract on umbilical cord blood mesenchymal stem cell expansion: Is there any need to save the amniotic membrane besides the umbilical cord blood? Iran J Basic Med Sci 19: 89-96, 2016.

18. Capobianco V, Caterino M, Iaffaldano L, Nardelli C, Sirico A Del Vecchio L, Martinelli P, Pastore L, Pucci P and Sacchetti L: Proteome analysis of human amniotic mesenchymal stem cells (hA-MSCs) reveals impaired antioxidant ability, cytoskeleton and metabolic functionality in maternal obesity. Sci Rep 6: 25270,2016

19. Zhou H, Zhang H, Yan Z and Xu R: Transplantation of human amniotic mesenchymal stem cells promotes neurological recovery in an intracerebral hemorrhage rat model. Biochem Biophys Res Commun 475: 202-208, 2016.
20. Herrmann RP and Sturm MJ: Adult human mesenchymal stromal cells and the treatment of graft versus host disease. Stem Cells Cloning 7: 45-52, 2014.

21. Belmar-Lopez C, Mendoza G, Oberg D, Burnet J, Simon C, Cervello I, Iglesias M, Ramirez JC, Lopez-Larrubia P, Quintanilla M, Martin-Duque P, et al: Tissue-derived mesenchymal stromal cells used as vehicles for anti-tumor therapy exert different in vivo effects on migration capacity and tumor growth. BMC Med 11: 139, 2013.

22. Sharma M, Afrin F, Tripathi R and Gangenahalli G: Regulated expression of CXCR4 constitutive active mutants revealed the up-modulated chemotaxis and up-regulation of genes crucial for CXCR4 mediated homing and engraftment of hematopoietic stem/progenitor cells. J Stem Cells Regen Med 9: 19-27, 2013.

23. Li J, Guo W, Xiong M, Han H, Chen J, Mao D, Tang B, Yu H and Zeng Y: Effect of SDF-1/CXCR4 axis on the migration of transplanted bone mesenchymal stem cells mobilized by erythropoietin toward lesion sites following spinal cord injury. Int J Mol Med 36: 1205-1214, 2015.

24. Díaz-Prado S, Muiños-López E, Hermida-Gómez T, Rendal-Vázquez ME, Fuentes-Boquete I, de Toro FJ and Blanco FJ: Multilineage differentiation potential of cells isolated from the human amniotic membrane. J Cell Biochem 111: 846-857, 2010.

25. Pelekanos RA, Ting MJ, Sardesai VS, Ryan JM, Lim YC, Chan JK and Fisk NM: Intracellular trafficking and endocytosis of CXCR4 in fetal mesenchymal stem/stromal cells. BMC Cell Biol 15: 15, 2014

26. Li Y, Yu X, Lin S, Li X, Zhang S and Song YH: Insulin-like growth factor 1 enhances the migratory capacity of mesenchymal stem cells. Biochem Biophys Res Commun 356: 780-784, 2007.

27. Li M, Yu J, Li Y, Li D, Yan D, Qu Z and Ruan Q: CXCR4 positive bone mesenchymal stem cells migrate to human endothelial cell stimulated by ox-LDL via SDF-1alpha/CXCR4 signaling axis. Exp Mol Pathol 88: 250-255, 2010.

28. Hung CM, Hsu YC, Chen TY, Chang CC and Lee MJ: Cyclophosphamide promotes breast cancer cell migration through CXCR4 and matrix metalloproteinases. Cell Biol Int 41: 345-352, 2017.

29. Sheng X, Zhong H, Wan H, Zhong J and Chen F: Granulocyte colony-stimulating factor inhibits CXCR4/SDF- $1 \alpha$ signaling and overcomes stromal-mediated drug resistance in the HL-60 cell line. Exp Ther Med 12: 396-404, 2016.

30. Shen W, Chen J, Zhu T, Chen L, Zhang W, Fang Z, Heng BC, Yin Z, Chen X, Ji J, et al: Intra-articular injection of human meniscus stem/progenitor cells promotes meniscus regeneration and ameliorates osteoarthritis through stromal cell-derived factor-1/CXCR4-mediated homing. Stem Cells Transl Med 3: 387-394, 2014. 